\title{
Effectiveness of Alpha-Fetoprotein Variants L2 and L3 as Substitutes of Alpha-Fetoprotein in Screening for Fetal Trisomy 18
}

Jianxia Huang

Hangzhou Hospital of Traditional Chinese Medicine

Yiming Chen

Hangzhou Women's Hospital

Wensheng Hu ( $\sim$ huwensheng010101@163.com )

hangzhou women's hospital https://orcid.org/0000-0001-5699-7717

Research article

Keywords: Trisomy 18; Alpha-fetoprotein variants L2 ;Alpha-fetoprotein Variants L3; screening

Posted Date: September 4th, 2019

DOI: https://doi.org/10.21203/rs.2.13899/v1

License: (c) (i) This work is licensed under a Creative Commons Attribution 4.0 International License. Read Full License 


\section{Abstract}

Purpose To evaluate the effectiveness of alpha-fetoprotein variants (AFP-L2, AFP-L3) for fetal screening of Trisomy 18 in place of alpha fetoprotein (AFP). Methods A case-control study was conducted. The case group included 39 pregnant women diagnosed by karyotype analysis of amniotic fluid cells as bearing Trisomy 18 fetuses. The control group included 48 pregnant women with clinically normal and healthy fetal development. The serum AFP-L2 and AFP-L3 concentrations were detected. Receiver operating characteristic (ROC) curves were used to determine the optimal cutoff value, area under the curve (AUC), and assess the screening performance of AFP-L2 and AFP-L3. Results The AFP-L2 and AFP-L3 concentrations in pregnant women with Trisomy 18 fetuses were $7.95 \pm 3.57 \mathrm{ng} / \mathrm{mL}$, and $2.53 \pm 1.80$ $\mathrm{ng} / \mathrm{mL}$, respectively, which was significantly higher than those of the control group $(3.73 \pm 1.63 \mathrm{ng} / \mathrm{mL}$ [3.26 4.20], $\mathrm{t}=6.820, \mathrm{P}<0.001$, and $0.84 \pm 0.60 \mathrm{ng} / \mathrm{mL}[0.66 \sim 1.01], \mathrm{t}=5.588, \mathrm{P}<0.001$, respectively). The AUC for AFP-L2 and AFP-L3 in screened Trisomy 18 fetuses was 0.848 (95\% Cl: 0.767-0.930, P < 0.001) and 0.806 (95\% Cl: $0.707-0.905, \mathrm{P}<0.001)$, respectively. The optimal cutoff values of AFP-L2 and AFP-L3 for Trisomy 18 fetuses were determined to be $6.340 \mathrm{ng} / \mathrm{mL}$ and $1.705 \mathrm{ng} / \mathrm{mL}$, respectively. The corresponding sensitivity, specificity, and Youden index values were $0.615,1.000,0.615 ; 0.6410,0.958$, and 0.599 , respectively. Comparisons across multiple modeling methods showed that the highest AUC of screened Trisomy 18 fetuses (0.992 and 0.986) was yielded by AFP-L2 + AFP-L3 + free $\beta$-HCG, and AFP-L2 + free $\beta$-HCG, with 1.000 sensitivity indicated in both instances. The sensitivities of the four Trisomy 18 screening combinations were all 1.000. In the control group, false positive rates of $22.92 \%, 8.33 \%, 12.50 \%$, and $12.50 \%$ were observed. The series test showed that the sensitivities of Trisomy 18 screening was $69.23 \%, 61.54 \%, 64.10 \%$, and $43.59 \%$, respectively, while a $3.28 \%$ false positive rate was found in the control group AFP + free $\beta$-HCG marker combination. Conclusion AFP-L2 and AFP-L3 showed high sensitivity and specificity in the screening of fetuses for Trisomy 18 . The new indicators did not bring about a significant improvement in screening efficiency but false positive rate was reduced compared to AFP.

\section{Introduction}

Trisomy 18, also known as Edwards syndrome (ES), is an autosomal trisomy syndrome whose incidence is second only to Trisomy 21 (Downs' syndrome, DS). Its typical clinical manifestations include physical abnormalities (including occipital kyphosis, narrow forehead, small head, and wide frontal suture, etc.), growth retardation, and heart and kidney defects. In order to be considered as Trisomy 18, the fetus needed to have an additional copy of all, or part of, chromosome 18. Children with chimeric trisomy 18 have milder clinical manifestations, varying degrees of developmental delay, and longer survival time. Children with a three-body malformation in the short arm of chromosome 18 show unobvious deformity which may manifest as stunting. The above characteristics can be used to perform a preliminarily screen for Trisomy 18, and a chromosome examination is required for the clinical diagnosis. The typical karyotypes are $47, X X,+18$ or $47, X Y,+18$. Its prevalence in live births is between $1 / 6000$ and $1 / 8000$ [1]. The average annual prevalence in the local area is 1.31/10 000 [2]. Trisomy 18 often precedes spontaneous abortion. With the development of prenatal diagnostic techniques in recent years, most 
Trisomy 18 fetuses are naturally eliminated or artificially terminated, so the incidence of Trisomy 18 is higher than its prevalence in live births reaching about 1/2 500ه1/2 600 [1]. Most children with Trisomy 18 die within 1 year of being born, and only $5 \%$ to $10 \%$ survive beyond this age [3]. The diagnosis of most Trisomy 18 cases is currently based on maternal age, maternal serum marker screening (alpha fetoprotein [AFP] + free beta human chorionic gonadotropin [free $\beta-H C G]$ ] [4], or ultrasound imaging $[5,6]$. It has been reported that AFP-L3 is superior to AFP in screening for Trisomy 21 [7-10], but there are no reports on the combined use of AFP-L2 and AFP-L3 as markers for Trisomy 18 screening. This case-control study selected 48 pregnant women with healthy fetuses and 39 pregnant women with a prenatal diagnosis of Trisomy 18 in the fetus to investigate the relationship between maternal serum AFP-L2 and AFP-L3 levels and Trisomy 18 fetuses. Additionally, as a potential replacement for AFP screening, the effectiveness of AFP-L2 and AFP-L3 combined with free $\beta$-HCG concentrations in screening for Trisomy 18 fetuses was tested.

\section{Objects And Methods}

Between October 2007 and September 2016 we used a case-control study method to analyze women pregnant with single fetuses that were $15 \varangle 20^{+6}$ weeks old. These women attended prenatal clinics associated with one of two prenatal screening centers [Hangzhou Women's Hospital (Hangzhou Maternity and Child Health Care Hospital), and the Maternity and Child Health Care Hospital, Yuhang District, Hangzhou, China]. We analyzed a total of 463298 cases, including 366355 cases of Hangzhou Women's Hospital (Hangzhou Maternity and Child Health Care Hospital) and 96943 cases in another hospital. Per the presence or absence of fetal Trisomy 18, the study subjects were divided into case and control groups, respectively. The case group included 39 pregnant women diagnosed by karyotype analysis of amniotic fluid as carrying Trisomy 18 fetuses, while the control group included 48 pregnant women with normal fetal development by choosing the next two chronologically consecutive cases after each Trisomy 18 case. All of them had an AFP and free $\beta$-HCG screening. The study was approved by the hospital ethics committee, and the approval number was [2018] medical ethics (004) No.01.

Exclusion criteria applied to both groups, including: 1) twins or at least one previous pregnancy; 2) the coexistence of other medical conditions such as insulin-dependent diabetes and severe pregnancy complications; 3 ) smoking; 4) pregnancy via in vitro fertilization; 5) follow-up results showing neural tube defects or other congenital birth defects; 6 ) cases with incomplete clinical or molecular data; and 7) a mismatch between maternal information and serum specimens.

Reagents and instruments: Human alpha-fetoprotein variants (AFP-L2 and AFP-L3) were detected using a RT-6100 microplate reader (Rayto, USA), 988 plate washers (Beijing Tianshi, China), and AFP-L2 and AFPL3 reagent (BIM, USA, batch numbers: B170658 and B160785, respectively).

Detection method: A total of $2 \varangle 3 \mathrm{ml}$ of peripheral venous blood was taken from fasting patients. Serum samples were separated and stored in a refrigerator at $2 \varangle 8^{\circ} \mathrm{C}$ within 30 minutes of collection. The samples were sent for analysis within 1 week of collection. Residual serum was stored at $-80^{\circ} \mathrm{C}$ after the prenatal 
screening test was performed. A double-antibody one-step ELISA method was used to detect AFP-L2 and AFP-L3 concentrations. The measured AFP, free $\beta$-HCG, AFP-L2, and AFP-L3 levels were expressed as multiples of the median (MoM).

\section{Statistical analyses}

Microsoft ${ }^{\circledR}$ Excel 2007 was used to establish a test result database which was processed by SPSS, version 21.0 software (SPSS, USA). A data normality test was performed using the one-sample Kolmogorov-Smirnov test, and the normally distributed data measured by the mean \pm standard deviation $( \pm s)$. AFP-L2 and AFP-L3 concentrations were compared between the case and control groups using two independent sample t-tests. The Trisomy 18 risk value was calculated by multiplying the age risk of Trisomy 18 [11] by the corresponding normal distribution likelihood [12]. The joint risk threshold is the log value of the corresponding risk value. Receiver operating characteristic (ROC) curves were plotted and the optimal cut-off, area under the curve (AUC), and Youden index values for AFP-L2 and AFP-L3 concentrations calculated accordingly. $P<0.05$ was considered significant.

\section{Results}

As shown in Table 1, there were no significant differences in mothers' age, pregnancy duration and body weight between the case and control group women.

Comparison of serum AFP-L2 and AFP-L3 levels in the two groups: As reflected in Table 2, the serum AFPL2 concentration in the case group was $7.95 \pm 3.57 \mathrm{ng} / \mathrm{mL}(6.79 \llbracket 9.10 \mathrm{ng} / \mathrm{mL})$, which was significantly higher than that of the control group $(3.73 \pm 1.63 \mathrm{ng} / \mathrm{mL}[3.26 \varangle 4.20 \mathrm{ng} / \mathrm{mL}], t=6.820, P<0.001)$. The serum AFP-L3 levels in the case group was $2.53 \pm 1.80 \mathrm{ng} / \mathrm{mL}(1.94 \llbracket 3.11 \mathrm{ng} / \mathrm{mL})$, which was significantly higher than that of the control group $(0.84 \pm 0.60 \mathrm{ng} / \mathrm{mL}[0.66 \otimes 1.01 \mathrm{ng} / \mathrm{mL}], t=5.588, P<0.001)$.

The value of AFP-L2 and AFP-L3 as independent indicators of Trisomy 18: Use of AFP-L2 for the screening of Trisomy 18 fetuses had an AUC of 0.848 ( $95 \% \mathrm{Cl}$ : $0.767 \rrbracket 0.930, P<0.001)$. Per the ROC curve, the optimal value of AFP-L2 for fetal Trisomy 18 screening was $6.340 \mathrm{ng} / \mathrm{ml}$. The sensitivity, specificity and Youden index values were $0.615,1.000$ and 0.615 , respectively. When screening Trisomy 18 fetuses using AFP-L3, the AUC was $0.806(95 \% \mathrm{Cl}$ : $0.707 ه 0.905, P<0.001)$ with the optimal AFP-L3 value determined to be $1.705 \mathrm{ng} / \mathrm{mL}$ following ROC analysis. The corresponding sensitivity, specificity and Youden index values were $0.641,0.958$ and 0.599 , respectively. The data are summarized in Table 3 and Figures 1 - 2.

Figure 1 AFP-L2 2AAFP-L3 single indicator screening ROC curve for trisomy 18. AFP-L2,a-fetoprotein heterogeneity L2; AFP-L3, a-fetoprotein heterogeneity L3

Figure 2 AFP $\square$ free $\beta$-HCG single indicator screening ROC curve for trisomy 18. AFP, a-fetoprotein; free $\beta$ HCG, free $\beta$-Human Chorionic Gonadotropin 
Value of the combined use of AFP-L2 and AFP-L3 markers when screening Trisomy 18 fetuses: Following comparisons between different modeling methods, it was shown that AFP-L $2+$ AFP-L3 + free $\beta$-HCG and AFP-L2 + free $\beta$-HCG had the highest AUC for screening Trisomy 18 fetuses, reaching 0.992 (95\% Cl: 0.981 $\varangle 1.000, P<0.001)$ and $0.986(95 \% \mathrm{Cl}: 0.967 \otimes 1.000, P<0.001)$, respectively. The sensitivity of both modes was $100 \%$ (Table 3 and Figure 3 ).

Figure 3 Multi-indicator joint screening ROC curve for trisomy 18. AFP, a-fetoprotein; free $\beta$-HCG, free $\beta$ Human Chorionic Gonadotropin; AFP-L2,a-fetoprotein heterogeneity L2; AFP-L3, a-fetoprotein heterogeneity L3

Comparison of screening Trisomy 18 fetuses using AFP-L2 + AFP-L3, and AFP + free $\beta$-HCG: In parallel test results, the sensitivity of AFP + free $\beta-H C G, A F P-L 2+$ free $\beta-H C G, A F P-L 3+$ free $\beta-H C G$, and AFP-L2 + AFP-L3 + free $\beta$-HCG in detecting Trisomy 18 were all 100\%, while in the control group, the four modes showed $22.92 \%, 8.33 \%, 12.50 \%$, and $12.50 \%$ false positive rates. In series test results, the sensitivity of AFP + free $\beta-H C G$, AFP-L2 + free $\beta-H C G, A F P-L 3+$ free $\beta-H C G$, and AFP-L2 + AFP-L3 + free $\beta-H C G$ in detecting Trisomy 18 was $69.23 \%, 61.54 \%, 64.10 \%$, and $43.59 \%$, respectively. In the control group, the AFP + free $\beta$-HCG mode showed a false positive rate of $3.28 \%$, while no instances of false positive calls were found in the three remaining screening modes (Table 4 ).

\section{Discussion}

Studies have shown that AFP-L2 and AFP-L3 are superior to AFP in detecting Trisomy 21 [7-10], but the joint use of AFP-L2 and AFP-L3 in screening for Trisomy 18 has not been investigated. Consequently, in order to explore the relationship between maternal serum AFP-L2 and AFP-L3 levels, as well as their screening efficiency for Trisomy 18, this case-control study included 39 pregnant women with a prenatal diagnosis of Trisomy 18, and 48 pregnant women with healthy fetuses. The results of this study showed that AFP-L2 and AFP-L3 serum levels are significantly higher in Trisomy 18 fetuses than in the control group $(P<0.01)$.

Yamamoto et al. [7] used a liquid phase binding assay to measure AFP concentration and AFP-L3\% in maternal serum. The AUC of AFP MoM, AFP-L3\%, AFP-L3 MoM, and AFP-L3 MoM/AFP MoM were 0.750, 0.868, 0.949, and 0.946, respectively. The AUC of AFP-L3 MoM and AFP-L3 MoM/AFP MoM was significantly higher than AFP-L3\% $(P<0.05)$ and AFP MoM $(P<0.05)$. Feng et al. [13] showed that the AUC of AFP-L3\% was 0.710 , and that Trisomy 21 bearing and normal pregnant women can be well distinguished when AFP-L3\% $\geq 10 \%$ is set as the standard. AFP-L2 and AFP-L3 screenings in this study had AUCs of 0.848 and 0.806 , respectively. Per the ROC curves, the optimal cutoff values of AFP-L2 and AFP-L3 for Trisomy 18 fetuses were $6.340 \mathrm{ng} / \mathrm{mL}$ and $1.705 \mathrm{ng} / \mathrm{mL}$, respectively. The sensitivity, specificity and Youden index values were $0.615,1.000,0.615$, and $0.641,0.958,0.599$, respectively. The results of this study show that both AFP-L2 and AFP-L3 are effective differentiators between pregnant women who bear healthy or Trisomy 18 fetuses. 
Our previous laboratory study has shown that AFP-L2 and AFP-L3 are good markers for the screening of Trisomy 21 fetuses in the second trimester of pregnancy due to high sensitivity and specificity. Screening with combined markers is better than screening with individual markers, while screening with AFP-L2 + AFP-L3 + free $\beta$-HCG showed the most effective results [14]. In the current study, different modeling methods were used to test Trisomy 18 screening results. The highest AUC for Trisomy 18 fetuses was yielded by AFP-L2 + AFP-L3 + free $\beta-H C G$ and AFP-L2 + free $\beta-H C G$, both of which had values of 0.992 and 0.986 with 1.000 sensitivity. This indicated that maternal serum AFP-L2 and AFP-L3 are good markers for Trisomy 18 as they have high sensitivity and specificity for screening of Trisomy 18 fetuses in the second trimester of pregnancy.

In this study, AFP-L2 and AFP-L3 were used as substitutes for AFP to screen for fetal Trisomy 18. Results of the two combined tests were compared. In the parallel test, the sensitivity of the four screening mode combinations in detecting Trisomy 18 was $100 \%$. In the control group, false positive rates of $22.92 \%$, $8.33 \%, 12.50 \%$, and $12.50 \%$ were observed. In the series test, Trisomy 18 detection sensitivity was $69.23 \%$, $61.54 \%, 64.10 \%$, and $43.59 \%$ in the control group, respectively. In the four screening modes of the control group, a $3.28 \%$ false positive rate was observed in the AFP + free $\beta$-HCG mode, while no false positive cases were observed in the three remaining screening modes. Likewise, Huai et al. [15] showed that Trisomy 21 screening using AFP-L3 instead of AFP was of higher sensitivity with a slightly higher false positive rate. Yamamoto et al. [16] showed that maternal blood and amniotic fluid had similar concentrations of AFP-L3. This was found not to affect the permeability of the membrane. AFP-L3\% in maternal serum can effectively distinguish whether or not a fetus has Trisomy 21 . With a false positive rate of $5 \%, 55 \%$ of pregnant women with serum AFP-L3\% were found to be carrying a fetus with Trisomy 21. AFP-L3 has been shown to be an effective alternative to AFP in prenatal Trisomy 21 screening. With high sensitivity and specificity, the results of this study indicated that AFP-L2 and AFP-L3 can also be used in combination with AFP for joint screening of Trisomy 18 fetuses.

In summary, with high sensitivity and specificity, AFP-L2 and AFP-L3 are good joint markers for Trisomy 18 screening when replacing the AFP marker. Although the screening efficiency is not significantly improved, it does significantly reduce the false positive rate when compared to AFP.

\section{Declarations}

\section{Ethics Approval and Consent to Participate}

The study was approved by Hangzhou Women's Hospital (Hangzhou Maternity and Child Health Care Hospital) ethics committee, in accordance with the ethical standards as laid down in the 1964 Declaration of Helsinki and its later amendments or comparable ethical standards. And the approval number was [2018] medical ethics (004) No.01. Consent you obtained from study participants was written.

\section{Consent for publication}




\section{Availability of data and material}

All data generated or analyzed during this study are included in this published article.

\section{Competing interests}

No competing interests were involved.

\section{Funding}

Natural Science Foundation of Zhejiang (GF19H040017); Hangzhou Science and Technology Plan Guidance Project(20181228Y13); Hangzhou Health Science and Technology Plan Project (2017A55) funded this study.

\section{Authors' Contributions}

Protocol/project development: all authors. Data collection: all authors. Revising and final approval: all authors. Methodology: JH, YC. Data analysis: JH, YC. Drafting: JH, YC, WH. All authors have read and approved the manuscript.

\section{Acknowledgements}

We thank Natural Science Foundation of Zhejiang (GF19H040017); Hangzhou Science and Technology Plan Guidance Project(20181228Y13); Hangzhou Health Science and Technology Plan Project (2017A55) funded this study, but had no role in study design, data gathering, data analysis, data interpretation. We thank Linyuan Gu and Xuelian Chu from the Maternity and Child Health Care Hospital in the Yuhang District of Hangzhou, and Shaolei Lv and Ran Xin from the Data Analytics Department of Zhejiang Bosheng Biotechnology Co. Ltd. for their contribution to specimen storage, case follow-up, data analyses, and modeling for this project.

\section{References}

1. CEREDA A, CAREY J C. The trisomy 18 syndrome [J]. Acta Ophthalmologica, 2012, 7(1):1-14.

2. Chen Yiming, Lu Sha, Zhang Wen, et al. The epidemiological study of fetal Edwards'syndrome in Hangzhou [J]. Preventive Medicine, 2018, 30(12):1284-1287. DOI:10.19485/j.cnki.issn20965087.2018.12.027 
3. BATEES H囚ALTIRKAWI KA. Trisomy 18 syndrome: Towards a balanced approach [J]. Sudan J Paediatr, 2014. 14(2): 76-84.

4. Baer R J, Flessel M C, Jelliffepawlowski $L L$, et al. Detection rates for aneuploidy by first-trimester and sequential screening [J]. Obstetrics \& Gynecology, 2015, 126(4):753-759.

DOI:10.1097/AOG.0000000000001040

5. Kroes I, Janssens S, Defoort P. Ultrasound features in trisomy 13 (Patau syndrome) and trisomy 18 (Edwards syndrome) in a consecutive series of 47 cases [J].Facts Views Vis Obgyn. 2014, 6(4):245249.

6. Naor D M, Maymon R, Keidar R, et al. [Prenatal diagnosis of trisomy 13 and trisomy 18: the experience of Assaf-Harofe Medical Center][J]. Harefuah, 2014, 153(8):453-457ه499, 498.

7. Yamamoto R, Azuma M, Wakui Y, et al. Alpha-fetoprotein microheterogeneity: a potential biochemical marker for Down's syndrome [J]. Clin Chim Acta, 2001. 304(1-2):137-141. DOIख10.1016/S00098981(00)00381-8

8. Yamamoto R, Minobe S, Ebina Y, et al. Prenatal trisomy 21 screening using the Lens culinaris agglutinin-reactive alpha-fetoprotein ratio[J]. Congenit Anom (Kyoto), 2004. 44(2): 87-92. DOI区 10.1111/j.1741-4520.2004.00013.x

9. Yu J, He LM, Hu HY, et al. Application of alpha-fetoprotein variant L2 in screening of Down's syndrome [J]. International Journal of Laboratory Medicine. 2015, 36(18): 2643-2644. DOIه 10.3969/j.issn.1673-4130.2015.18.011

10. Wu H, Zhang YS, Wu XX, et al. The value of alpha-fetoprotein heterogeneity in screening of Down's syndrome [J]. Chinese Journal of Birth Health \& Heredity. 2012(02): 56-57.

11. Cuckle H S, Wald N J, Thompson S G. Estimating a woman's risk of having a pregnancy associated with Down's syndrome using her age and serum alpha-fetoprotein level[J]. BJOG: An International Journal of Obstetrics \& Gynaec ology, 1987, 94(5): 387-402. Dolه10.1016/0020-7292(88)90310-4

12. Royston $P$, Thompson $S \mathrm{G}$. Model-based screening by risk with application to Down's syndrome [J]. Statistics in Medicine, 1992, 11(2): 257-268. Dolه10.1002/sim.4780110211

13. Long F, Duan C, Liu JN, et al. Clinical significance of alpha-fetoprotein heterogeneity in pregnant metaphase in Down's syndrome screening [J]. Chinese Journal of the Frontiers of Medical Science (Electronic Version). 2014, 6(3): 43-45. DOlه10.3969/j.issn.1674-7372.2014.03.016

14. Chen Yiming, Lu Sha, Lian Jiejing, et al. Clinical value of maternal serum alpha-fetoprotein heterogeneity L2 and L3 in second trimester in fetal Down's syndrome screening[J]. Zhejiang Medical Journal. 2019, 41(1): 27-30+39. DOIه10.12056/j.issn.1006-2785. 2019.41.1. 2018-1532

15. Huai L, Leng J, Ma S, et al. Replacing alpha-fetoprotein with alpha-fetoprotein-L3 increases the sensitivity of prenatal screening for trisomy 21[J]. American Journal of Therapeutics, 2016, 23(6): e1754-e1757. DOIه10.1097/MJT.0000000000000418

16. Yamamoto R, Azuma M, Hoshi N, et al. Lens culinaris agglutinin-reactive a-fetoprotein,an alternative variant to a-fetoprotein in prenatal screening for Down's syndrome[J]. Human Reproduction, 2001, 16(11): 2438-2444. DOIه10.1093/humrep/16.11.2438 
Table 1 Comparison of baseline data of each group

\begin{tabular}{ccccc}
\hline Group & $\mathrm{n}$ & Age $\llbracket$ year $\square$ & Days of pregnancy $\square \mathrm{d} \square$ & Weight $\square \mathrm{kg} \square$ \\
\hline trisomy 18 & 39 & $28.22 \pm 3.72(27.02 \square$ & $118.38 \pm 6.35(116.33 \square$ & $50.05 \pm 7.76(53.53 \square$ \\
group & & $29.43)$ & $120.44)$ & $58.56)$ \\
Control group & 48 & $28.75 \pm 2.94(29.90 \square$ & $117.52 \pm 4.52(116.21 \square$ & $56.42 \pm 7.79(54.16 \square$ \\
$t$ & - & $29.60)$ & $118.83)$ & $58.68)$ \\
$P$ & - & 0.737 & 0.740 & 0.222 \\
& -0.463 & 0.461 & 0.825 \\
\hline
\end{tabular}

Table 2 Comparison of serum AFP-L2 and AFP-L3 levels in the two groups

\begin{tabular}{|c|c|c|c|c|c|}
\hline Group & $\mathrm{n}$ & $\mathrm{AFP}[\mathrm{U} / \mathrm{mL}]$ & $\begin{array}{l}\text { free } \beta-H C G \\
{[\mathrm{ng} / \mathrm{ML}]}\end{array}$ & $\begin{array}{l}\text { AFP-L2 } \\
{[\mathrm{ng} / \mathrm{mL}]}\end{array}$ & $\begin{array}{c}\text { AFP-L3 } \\
{[\mathrm{ng} / \mathrm{mL}]}\end{array}$ \\
\hline trisomy & 39 & $21.57 \pm 9.86(18.38 \square$ & $2.73 \pm 1.34(2.29 \square$ & $7.95 \pm 3.57(6.79$ & $2.53 \pm 1.80$ \\
\hline 18 group & & 24.77) & 3.16) & प9.10) & ૧3.11) \\
\hline $\begin{array}{l}\text { Control } \\
\text { group }\end{array}$ & 48 & $\begin{array}{l}34.16 \pm 14.69(29.89 \\
\square 38.42)\end{array}$ & $\begin{array}{l}46.20 \pm 23.56(39.36 \\
\square 53.04)\end{array}$ & $\begin{array}{c}3.73 \pm 1.63(3.26 \\
\square 4.20)\end{array}$ & $\begin{array}{l}0.84 \pm 0.60(0.66 \\
\square 1.01)\end{array}$ \\
\hline$t$ & - & 4.576 & 12.758 & 6.820 & 5.588 \\
\hline$P$ & - & $<0.001$ & $<0.001$ & $<0.001$ & $<0.001$ \\
\hline
\end{tabular}

AFP, $\alpha$-fetoprotein; free $\beta$-HCG, free $\beta$-Human Chorionic Gonadotropin; AFP-L2, $\alpha$-fetoprotein heterogeneity 2; AFP-L3, $\alpha$-fetoprotein heterogeneity L3;

Table 3 Value of AFP $\triangle A F P-L 2 \square A F P-L 3$ Separate screening and joint screening for the Trisomy 18

\begin{tabular}{|c|c|c|c|c|c|c|c|}
\hline $\begin{array}{l}\text { Screening } \\
\text { method }\end{array}$ & AUC & $95 \% \mathrm{CI}$ & $P$ & $\begin{array}{l}\text { Cut } \\
\text { off }\end{array}$ & Sensitivity & Specificity & $\begin{array}{l}\text { Youden } \\
\text { index }\end{array}$ \\
\hline $\begin{array}{l}\text { free } \beta \text { - } \\
\text { HCG }\end{array}$ & 0.962 & $\begin{array}{l}0.923 \square \\
1.000\end{array}$ & $<0.001$ & 11.325 & 0.917 & 1.000 & 0.917 \\
\hline $\mathrm{AFP}$ & 0.826 & $\begin{array}{l}0.739 \\
0.914\end{array}$ & $<0.001$ & 22.600 & 0.812 & 0.692 & 0.505 \\
\hline AFP-L2 & 0.848 & $\begin{array}{l}0.767 \square \\
0.930\end{array}$ & $<0.001$ & 6.340 & 0.615 & 1.000 & 0.615 \\
\hline AFP-L3 & 0.806 & $\begin{array}{l}0.707 \square \\
0.905\end{array}$ & $<0.001$ & 1.705 & 0.641 & 0.958 & 0.599 \\
\hline $\begin{array}{c}\text { AFP+ } \\
\text { free } \beta-H C G\end{array}$ & 0.967 & $\begin{array}{l}0.932 \square \\
1.000\end{array}$ & $<0.001$ & 5.138 & 1.000 & 0.917 & 0.967 \\
\hline $\begin{array}{l}\text { AFP-L2+ } \\
\text { tree } \beta-H C G\end{array}$ & 0.986 & $\begin{array}{l}0.967 \square \\
1.000\end{array}$ & $<0.001$ & 4.150 & 1.000 & 0.938 & 0.938 \\
\hline $\begin{array}{l}\text { AFP-L3+ } \\
\text { tree } \beta-H C G\end{array}$ & 0.976 & $\begin{array}{l}0.948 \square \\
1.000\end{array}$ & $<0.001$ & 4.976 & 1.000 & 0.929 & 0.929 \\
\hline $\begin{array}{c}\text { AFP- } \\
\text { 2+AFP-L3+ } \\
\text { free } \beta \text {-HCG }\end{array}$ & 0.992 & $\begin{array}{l}0.981 \square \\
1.000\end{array}$ & $<0.001$ & 3.021 & 1.000 & 0.933 & 0.992 \\
\hline
\end{tabular}


Table 4 Comparison of four screening modes in two combined trials

\begin{tabular}{|c|c|c|c|c|c|}
\hline $\begin{array}{l}\text { Screening } \\
\text { mode }\end{array}$ & $\mathrm{n}$ & $\begin{array}{l}\text { AFP+free } \\
\beta \text {-HCG }\end{array}$ & $\begin{array}{l}\text { AFP-L2+free } \\
\beta-H C G\end{array}$ & $\begin{array}{l}\text { AFP-L3+free } \\
\beta-H C G\end{array}$ & $\begin{array}{l}\text { AFP-L2+AFP- } \\
\text { L3+free } \beta-H C G\end{array}$ \\
\hline $\begin{array}{l}\text { Parallel } \\
\text { test }\end{array}$ & & & & & \\
\hline $\begin{array}{l}\text { Trisomy } 18 \\
\text { group }\end{array}$ & 39 & $39(100 \%)$ & $39(100 \%)$ & $39(100 \%)$ & $39(100 \%)$ \\
\hline $\begin{array}{l}\text { Control } \\
\text { group } \\
\text { Series test }\end{array}$ & 48 & 14(22.92\%) & $5(8.33 \%)$ & $7(12.50)$ & $7(12.50)$ \\
\hline $\begin{array}{l}\text { Trisomy } 18 \\
\text { group }\end{array}$ & 39 & $27(69.23 \%)$ & $24(61.54)$ & $25(64.10 \%)$ & 17(43.59\%) \\
\hline $\begin{array}{l}\text { Control } \\
\text { group }\end{array}$ & 48 & $2(3.28 \%)$ & 0 & 0 & 0 \\
\hline
\end{tabular}

\section{Figures}

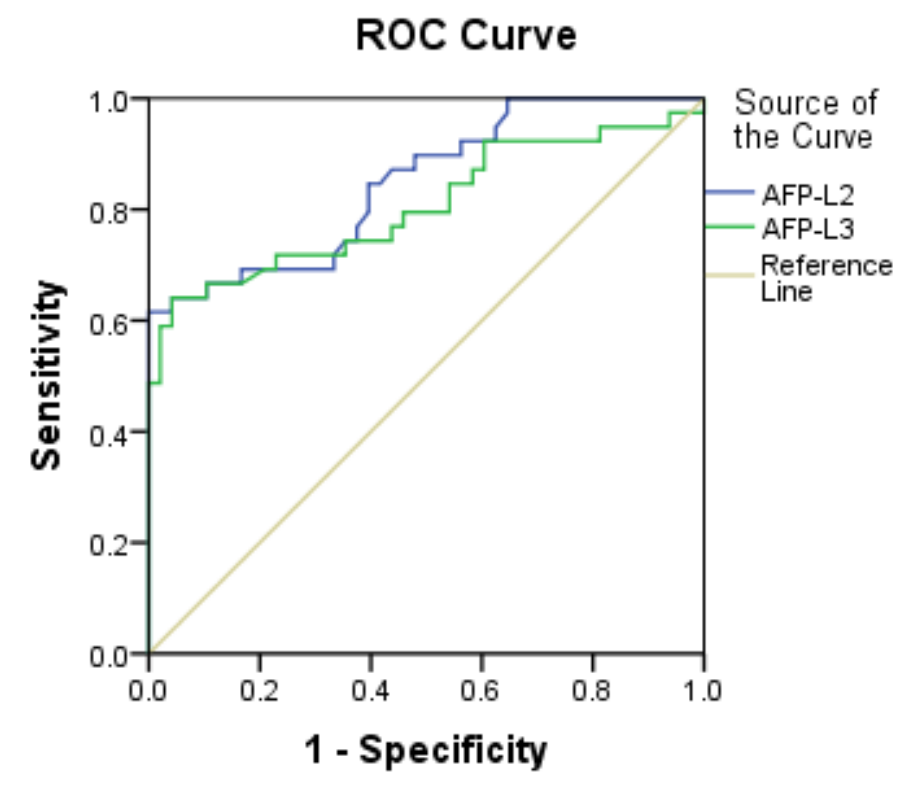

Figure 1

AFP-L2 2AFP-L3 single indicator screening ROC curve for trisomy 18. AFP-L2,a-fetoprotein heterogeneity L2; AFP-L3, a-fetoprotein heterogeneity L3 


\section{ROC Curve}



Figure 2

$A F P \square$ free $\beta$-HCG single indicator screening ROC curve for trisomy 18. AFP, a-fetoprotein; free $\beta$-HCG, free $\beta$ Human Chorionic Gonadotropin 


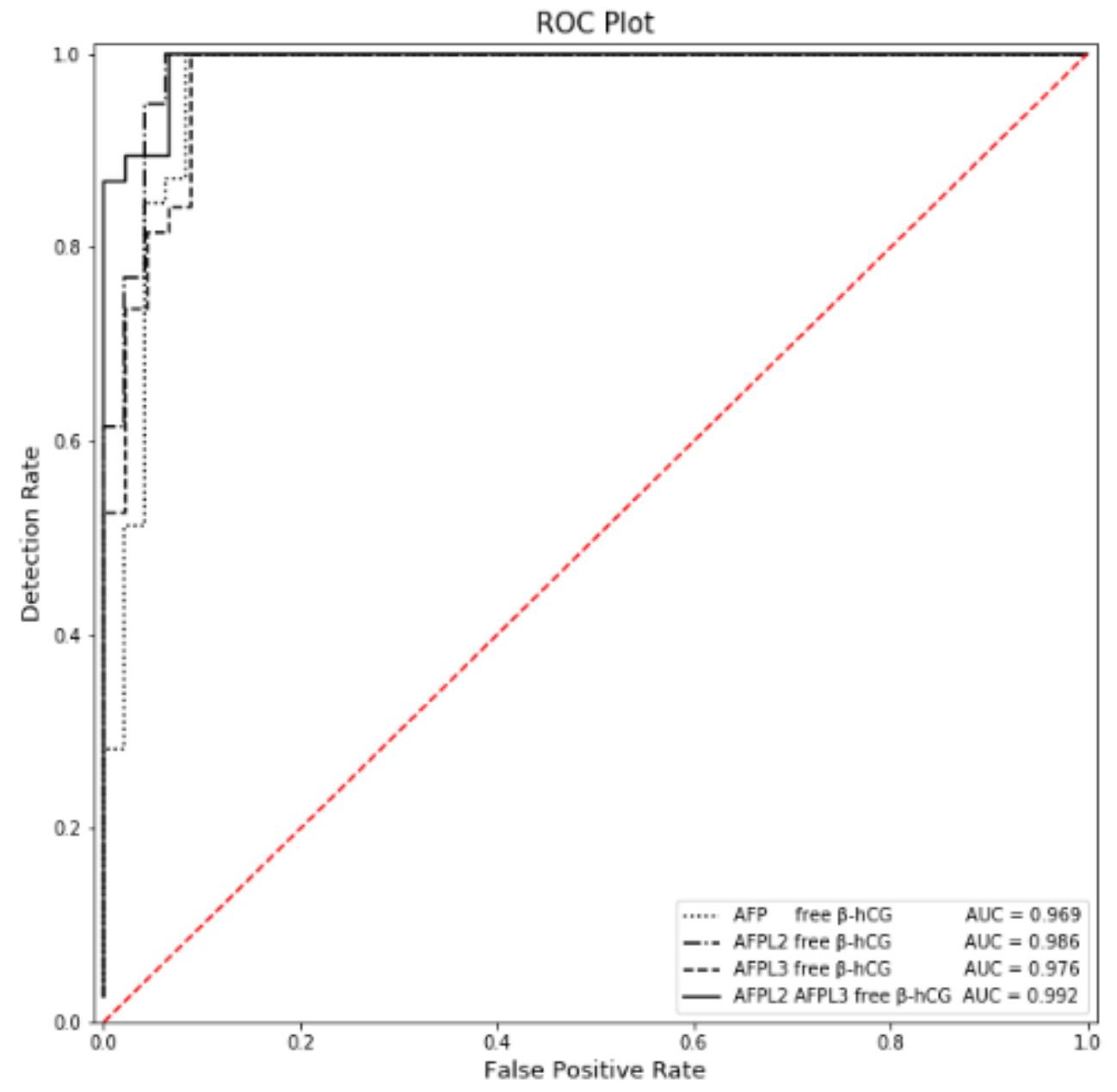

Figure 3

Multi-indicator joint screening ROC curve for trisomy 18. AFP, a-fetoprotein; free $\beta$-HCG, free $\beta$-Human Chorionic Gonadotropin; AFP-L2,a-fetoprotein heterogeneity L2; AFP-L3, a-fetoprotein heterogeneity L3 